\section{LIGHT MANIPULATION}

\section{Winding light beams}

Phys. Rev. A 94, 013829 (2016)

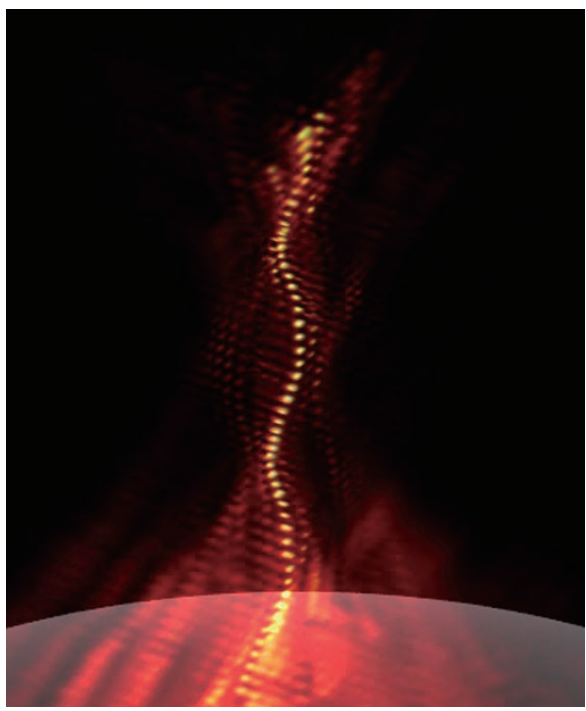

Caustic methods, which rely on phase manipulation and specific initial field distributions, make it possible to generate light trajectories other than straight lines, but so far most approaches have been limited to convex trajectories such as parabolae. Now, Yuanhui Wen and collaborators report the observation of light beams following arbitrary elliptical helical trajectories in free space. The key to their approach, based on a caustic technique that generates curved trajectories by interfering plane waves, was to consider a non-convex trajectory as a sequence of convex segments. To achieve the necessary phase and amplitude modulation of an incident light beam, the team fabricated a 0.5 -mm-thick square quartz glass plate with a side length of $20 \mathrm{~mm}$ comprising a smaller effective area divided into $601 \times 601$ pixels.
Each pixel was etched to determine a given phase shift, and a partial metal coverage realized specific transmittance patterns to modulate the beam amplitude.

$G D$

\section{FIBRE LASERS}

\section{Triwavelength source}

Appl. Phys. Express 9, 092701 (2016)

Mode-locked multiwavelength fibre lasers are useful for numerous applications ranging from wavelength-division multiplexing to bioimaging. In such systems, saturable absorbers are often employed within the laser cavity as a mode-locking element but shortcomings can include limited bandwidth or small optical modulation depth. Now, Ruwei Zhao and colleagues from China have demonstrated a triwavelength synchronously mode-locked erbium-doped fibre laser that uses black phosphorus as a saturable absorber. Stable operation was achieved with lasing wavelengths of $1,557.2,1,557.7$ and $1,558.2 \mathrm{~nm}$, and pulse repetition rate and duration of $1.65 \mathrm{MHz}$ and 9.41 ps, respectively. To make the saturable absorber, eight layers of black phosphorus from solution were deposited onto an erbium-doped fibre core. Pumping the device with $80 \mathrm{~mW}$ of power resulted in stable triwavelength lasing, whereas pumping beyond $170 \mathrm{~mW}$ disrupted mode-locking. By comparing devices with and without the black phosphorus, the team showed that its inclusion not only provided the mode-locking but also aided generation and stability of the three lasing wavelengths. Without the black phosphorus, homogeneous gain broadening in the erbium-doped fibre resulted in unstable multiwavelength oscillation. By contrast, over 30 min stable operation was demonstrated with the black phosphorus. $D P$
HOLOGRAPHY

\section{Optical forces for data storage}

Photorefractive media cannot store holographic information permanently as the photoinduced change in refractive index fades away with time. Now, Ali Yetisen and colleagues from the USA and the UK have developed robust and rewritable 3D holography that relies on optical forces instead. The recording medium was composed of poly(2hydroxyethyl methacrylate-co-methacrylic acid) ( $($ HEMA-co-MAA)) and silver metal nanoparticles ( $\mathrm{Ag}^{0} \mathrm{NPs}$ ). A glass slide coated with a 10- $\mu$ m-thick $p$ (HEMA-co-MAA) film containing $1 \mathrm{vol} \% \mathrm{Ag}^{0} \mathrm{NPs}$ was placed on a plane mirror at an angle from the mirror surface. A Nd:YAG laser provided a reference beam that propagated through the glass slide and was further reflected by the plane mirror. The interference between the reference beam and its reflection off the mirror created high- and low-intensity regions in the recording medium. Optical forces originating from this intensity gradient directed the $\mathrm{Ag}^{0} \mathrm{NPs}$ toward regions of minimum intensity, organizing them into reconfigurable 3D assemblies. The process of hologram recording was fully reversible, and holograms could be erased by making the glass slide parallel to the mirror.

\section{SEMICONDUCTOR SOURCES \\ High-power disk laser \\ Optica 3, 844-852 (2016)}

For semiconductor disk lasers there is a trade-off between the shortest pulse duration and the highest achievable pulse peak power. Now, Dominik Waldburger and colleagues from ETH Zürich in Switzerland have designed a vertical external-cavity surface-emitting laser (VECSEL) that uses a semiconductor saturable absorber mirror (SESAM) for mode-locking and offers significantly improved performance. The device emits 96-fs-long pulses (with external compression) at a rate of $1.6 \mathrm{GHz}$ and a peak power of $560 \mathrm{~W}$. The laser featured a simple V-shaped cavity with the output coupler and the SESAM at each end. The laser was optically pumped with $21 \mathrm{~W}$ of power from an 808-nm diode laser. The layer structures of the GaAs-based VECSEL were designed to have a large gain saturation fluence and a slightly positive but flat group delay dispersion over a large gain bandwidth. Numerical simulations suggest that the optical spectrum could support transform-limited pulses as short as $65 \mathrm{fs}$ if higher-order dispersion compensation was employed.

\section{D MATERIALS}

\section{Solar purification}

Nat. Nanotech. http://doi.org/bp75 (2016)

Films made from few-layered vertically aligned sheets of the popular 2D material molybdenum disulfide $\left(\mathrm{MoS}_{2}\right)$ topped with a 5-nm-thick layer of copper can act as a highly effective solar-based water purifier, according to scientists in California. Chong Liu and co-workers from Stanford University report that the films act as a photocatalyst, harvesting visible light to generate electron-hole pairs that react with water to produce bacteria-killing reactive oxygen species. Experimental tests indicate that water disinfection takes just a few minutes, with $99.999 \%$ of bacteria in water being inactivated in $20 \mathrm{~min}$ when $1.6 \mathrm{mg} \mathrm{l}^{-1}$ of the material is added to a water sample that is then exposed to visible light. Unlike other light-based purification schemes, the approach can work with visible rather than ultraviolet light. This is made possible by increasing the bandgap of $\mathrm{MoS}_{2}$ from $1.3 \mathrm{eV}$ (bulk material) to $1.55 \mathrm{eV}$ (few-layered $\mathrm{MoS}_{2}$ ). 